\title{
Cyclic cosmology from Lagrange-multiplier modified gravity
}

\author{
Yi-Fu Cai ${ }^{1,2, *}$ and Emmanuel N. Saridakis, \\ 1 Department of Physics, Arizona State University, Tempe, AZ 85287, USA \\ ${ }^{2}$ Institute of High Energy Physics, Chinese Academy of Sciences, P.O. Box 918-4, Beijing 100049, China \\ ${ }^{3}$ College of Mathematics and Physics, \\ Chongqing University of Posts and Telecommunications, Chongqing 400065, China
}

\begin{abstract}
We investigate cyclic and singularity-free evolutions in a universe governed by Lagrange-multiplier modified gravity, either in scalar-field cosmology, as well as in $f(R)$ one. In the scalar case, cyclicity can be induced by a suitably reconstructed simple potential, and the matter content of the universe can be successfully incorporated. In the case of $f(R)$-gravity, cyclicity can be induced by a suitable reconstructed second function $f_{2}(R)$ of a very simple form, however the matter evolution cannot be analytically handled. Furthermore, we study the evolution of cosmological perturbations for the two scenarios. For the scalar case the system possesses no wavelike modes due to a dust-like sound speed, while for the $f(R)$ case there exist an oscillation mode of perturbations which indicates a dynamical degree of freedom. Both scenarios allow for stable parameter spaces of cosmological perturbations through the bouncing point.
\end{abstract}

PACS numbers: 98.80.-k, 04.50.Kd

\section{INTRODUCTION}

Inflation is now considered to be a crucial part of the cosmological history of the universe [1], however the so called "standard model of the universe" still faces the problem of the initial singularity. Such a singularity is unavoidable if inflation is realized using a scalar field while the background spacetime is described by the standard Einstein action [2]. As a consequence, there has been a lot of effort in resolving this problem through quantum gravity effects or effective field theory techniques.

A potential solution to the cosmological singularity problem may be provided by non-singular bouncing cosmologies [3]. Such scenarios have been constructed through various approaches to modified gravity, such as the Pre-Big-Bang [4] and the Ekpyrotic [5] models, gravity actions with higher order corrections []. , braneworld scenarios [7], non-relativistic gravity [8], loop quantum cosmology [9] or in the frame of a closed universe [10]. Non-singular bounces may be alternatively investigated using effective field theory techniques, introducing matter fields violating the null energy condition [11] leading to observable predictions too [12], or introduce nonconventional mixing terms [13]. The extension of all the above bouncing scenarios is the (old) paradigm of cyclic cosmology [14], in which the universe experiences the periodic sequence of contractions and expansions, which has been rewaked the last years [15] since it brings different insights for the origin of the observable universe [16 18 ] (see [19] for a review).

On the other hand, the interesting idea of modifying gravity using Lagrange multipliers, first introduced in

\footnotetext{
*Electronic address: ycai21@asu.edu
}

† Electronic address: msaridak@phys.uoa.gr
[3] with bouncing solution studied in [20], was recently gained new interest, since its extended version may reduce irrelevant degrees of freedom in modified gravity models [21 23]. In particular, using two scalar fields, one of which is the Lagrange multiplier, leads to a constraint of special form on the second scalar field, and as a result the whole system contains a single dynamical degree of freedom.

In the present work we are interested in constructing a scenario of cyclic cosmology in universe governed by modified gravity with Lagrange multipliers. We do that both in the case of scalar cosmology, as well as in the case of $f(R)$-gravity, both in their Lagrange-multiplier modified versions. As we show, the realization of cyclicity and the avoidance of singularities is straightforward.

This paper is organized as follows. In section 【1 we present modified gravity with Lagrange multipliers, separately for scalar cosmology (subsection IIA) and for $f(R)$-gravity (subsection [IB). In section III we construct the scenario of cyclicity realization, reconstructing the required potential (subsection $\amalg I \mathrm{~A}$ ) and the form of $f(R)$ modification (subsection IIIB). In section IV we investigate the stability of the cosmological perturbations of the two scenarios. Finally, section $\mathrm{V}$ is devoted to the summary of our results.

\section{MODIFIED GRAVITY WITH LAGRANGE MULTIPLIERS}

Let us present the cosmological scenarios with Lagrange multipliers. In order to be general and complete, we present both the scalar case, as well as the $f(R)$ gravity one. Throughout the work we consider a flat Friedmann-Robertson-Walker geometry with metric

$$
d s^{2}=-d t^{2}+a^{2}(t) d \mathbf{x}^{2}
$$


where $a$ is the scale factor, although we could straightforwardly generalize our results to the non-flat case too.

\section{A. Scalar cosmology with Lagrange multiplier}

In this subsection, we consider a scenario with two scalars, namely $\phi$ and $\lambda$, where the second scalar is a Lagrange multiplier which constrains the field equation of the first one. The action reads [21, 222]:

$$
\begin{aligned}
& S=\int d^{4} x \sqrt{-g}\left\{\frac{R}{2 \kappa^{2}}-\frac{\omega(\phi)}{2} \partial_{\mu} \phi \partial^{\mu} \phi-V(\phi)\right. \\
&\left.-\lambda\left[\frac{1}{2} \partial_{\mu} \phi \partial^{\mu} \phi+U(\phi)\right]+\mathcal{L}_{m}\right\},
\end{aligned}
$$

where $\lambda$ is the Lagrange multiplier field. Furthermore, $V(\phi)$ and $U(\phi)$ are potentials of $\phi$, while the function $\omega(\phi)$ and especially its sign determines the nature of the scalar field $\phi$, that is if it is canonical or phantom. Finally, the term $\mathcal{L}_{m}$ accounts for the matter content of the universe.

Variation over the $\lambda$-field leads to

$$
0=\frac{\dot{\phi}^{2}}{2}-U(\phi)
$$

As we observe, this equation acts as a constraint for the other scalar field $\phi$, and it is the cause of the significant cosmological implications of such a construction. Now, the Friedmann equations straightforwardly write

$$
\begin{aligned}
\frac{3}{\kappa^{2}} H^{2} & =\left[\frac{\omega(\phi)+\lambda}{2}\right] \dot{\phi}^{2}+V(\phi)+\lambda U(\phi) \\
= & {[\omega(\phi)+2 \lambda] U(\phi)+V(\phi)+\rho_{m} }
\end{aligned}
$$

where we have defined the Hubble parameter as $H \equiv \frac{\dot{a}}{a}$, and we have also made use of the constraint (3). Additionally, in these expressions $\rho_{m}$ and $p_{m}$ stand respectively for the energy density and pressure of matter, with equation-of-state parameter $w_{m} \equiv p_{m} / \rho_{m}$. Observing the above equations, we can straightforwardly define the energy density and pressure for the dark-energy sector as

$$
\begin{aligned}
& \rho_{d e} \equiv[\omega(\phi)+2 \lambda] U(\phi)+V(\phi), \\
& p_{d e} \equiv \omega(\phi) U(\phi)-V(\phi),
\end{aligned}
$$

and thus the dark energy equation-of-state parameter will be

$$
w_{d e}=\frac{\omega(\phi) U(\phi)-V(\phi)}{[\omega(\phi)+2 \lambda] U(\phi)+V(\phi)} .
$$

Let us now explore some general features of the scenario at hand following [22]. First of all the constraint equation (3) can be integrated for positive $U(\phi)$, leading to

$$
t= \pm \int^{\phi} \frac{d \phi}{\sqrt{2 U(\phi)}}
$$

Inverting this relation with respect to $\phi$, one can find the explicit $t$-dependence of $\phi$, that is the corresponding $\phi(t)$. Thus, substituting the expression of $\phi(t)$ into (5), we obtain a differential equation for $H(t)$ :

$$
\begin{aligned}
2 \dot{H}(t)+3[H(t)]^{2}= & -\kappa^{2}\{\omega(\phi(t)) U(\phi(t)) \\
& \left.-V(\phi(t))+w_{m} \rho_{m}(t)\right\},
\end{aligned}
$$

the solution of which determines completely the cosmological evolution. Finally, substituting $\phi(t)$ and $H(t)$ into (41), we can find the $t$-dependence of the Lagrange multiplier field $\lambda$ :

$$
\begin{array}{r}
\lambda(t)=\frac{1}{2 U(\phi(t))}\left\{\frac{3}{\kappa^{2}} H(t)^{2}-V(\phi(t))-\rho_{m}(t)\right\} \\
-\frac{\omega(\phi(t))}{2} .
\end{array}
$$

However, one could alternatively follow the inverse procedure, that is to first determine the specific behavior of $H(t)$ and try to reconstruct the corresponding potential $V(\phi)$, which is responsible for the $\phi$-evolution that leads to such a $H(t)$. In particular, he first chooses a suitable $U(\phi)$ which will lead to an easy integration of (9), allowing for an interchangeable use of $\phi$ and $t$ through $\phi(t) \leftrightarrow t(\phi)$. Therefore, the second Friedmann equation (5) gives straightforwardly

$$
\begin{aligned}
V(\phi)=\frac{1}{\kappa^{2}}[2 \dot{H}(t(\phi)) & \left.+3 H(t(\phi))^{2}\right] \\
& +\omega(\phi) U(\phi)+w_{m} \rho_{m}(t(\phi)),
\end{aligned}
$$

where $\omega(\phi)$ can still be arbitrary. In summary, such a potential $V(\phi)$ produces a cosmological evolution with the chosen $H(t)$.

Finally, let us make a comment here concerning the nature of models with a second, Lagrange-multiplier field, since it is obvious that the constraint (31) changes completely the dynamics, comparing to the conventional models. In principle, one expects to have a propagation mode for each new field. However, due to the constraint (3) and the form in which the $\lambda$-field appears in the action, the propagating modes of $\phi$ and $\lambda$ do not appear, and the system is driven by a system of two first-order ordinary differential equations, one for each field. As a consequence, there are no propagating wave-like degrees of freedom and the sound speed for perturbations is exactly zero irrespective of the background solution [21].

\section{B. $f(R)$-gravity with Lagrange multiplier}

In this subsection we present $f(R)$-gravity with Lagrange multipliers, following [22]. In this case, we start 
by a conventional $f(R)$-gravity, and we add a scalar field $\lambda$ which is a Lagrange multiplier leading to a constraint. In particular, the action reads:

$$
S=\int d^{4} x \sqrt{-g}\left\{f_{1}(R)-\lambda\left[\frac{1}{2} \partial_{\mu} R \partial^{\mu} R+f_{2}(R)\right]\right\}
$$

where $f_{1}(R)$ and $f_{2}(R)$ are two independent functions of the Ricci scalar $R$. Note that in the above action we have not included the matter content of the universe, since this would significantly modify the multiplying terms of $\lambda$, making the subsequent reconstruction procedures tech- nically very complicated. These subtleties are caused by the fact that the Lagrange multiplier field propagates in this case, which is a disadvantage of the present scenario, contrary to the scalar cosmology of the previous subsection.

Variation over $\lambda$ leads to the constraint

$$
0=-\frac{1}{2} \dot{R}^{2}+f_{2}(R)
$$

Additionally, varying over the metric $g_{\mu \nu}$ and keeping the $(0,0)$-component we obtain

$$
0=-\frac{1}{2} f_{1}(R)+18 \lambda(\ddot{H}+4 H \dot{H})^{2}+\left\{3\left(\dot{H}+H^{2}\right)-3 H \frac{d}{d t}\right\}\left\{f_{1, R}-\lambda f_{2, R}+\left(\frac{d}{d t}+3 H\right)\left(\lambda \frac{d R}{d t}\right)\right\}
$$

where ${ }_{R}$ denotes the derivative with respect to the Ricci scalar.

For $f_{2}(R)>0$, the constraint (14) can be solved as

$$
t=\int^{R} \frac{d R}{\sqrt{2 f_{2}(R)}}
$$

and inverting this relation with respect to $R$ one can obtain the explicit $t$-dependence of $R$, that is the corresponding $R(t)$. On the other hand, the Ricci scalar is given from $R=6 \dot{H}+12 H^{2}$. Thus, inserting $R(t)$ in this relation one obtains a differential equation in terms of $H(t)$, namely

$$
6 \dot{H}(t)+12[H(t)]^{2}=R(t),
$$

the solution of which determines completely the cosmological behavior. Finally, using the obtained $H(t)$ and $R(t)$, equation (15) becomes a differential equation for the Lagrange multiplier field $\lambda(t)$ which can be solved.

However, one could alternatively follow the inverse procedure, that is to first determine the specific behavior of $H(t)$ and try to reconstruct the corresponding $f_{2}(R)$, which is responsible for the $R$-evolution that leads to such an $H(t)$. In particular, with a known $H(t)$ (17) provides immediately $R(t)$, which can be inverted, giving $t=t(R)$. Thus, using the constraint (14), the explicit form of $f_{2}(R)$ is found to be

$$
f_{2}(R)=\left.\frac{1}{2}\left(\frac{d R}{d t}\right)^{2}\right|_{t=t(R)}
$$

It is interesting to mention that in the above discussion $f_{1}(R)$ is arbitrary. That is, while in conventional $f(R)$ gravity the cosmological behavior is determined completely by $f_{1}(R)$, in Lagrange-multiplier modified $f(R)$ gravity the dynamics is determined completely by $f_{2}(R)$. In this case $f_{1}(R)$ would become relevant only in the presence of matter, and its effect on Newton's law [22].

\section{COSMOLOGICAL BOUNCE AND CYCLIC COSMOLOGY}

Having presented the cosmological models with Lagrange multipliers, both in the scalar, as well as in the $f(R)$-gravity case, in this section we are interested in investigating the bounce and cyclic solutions.

In principle, in order to acquire a cosmological bounce, one has to have a contracting phase $(H<0)$, followed by an expanding phase $(H>0)$, while at the bounce point we have $H=0$. In this whole procedure the timederivative of the Hubble parameter must be positive, that is $\dot{H}>0$. On the other hand, in order for a cosmological turnaround to be realized, one has to have an expanding phase $(H>0)$ followed by a contracting phase $(H<0)$, while at the turnaround point we have $H=0$, and in this whole procedure the time-derivative of the Hubble parameter must be negative, that is $\dot{H}<0$. Observing the form of Friedmann equations (4), (5), as well as of (15), we deduce that such a behavior can be easily obtained in principle. In the following two subsections we proceed to the detailed investigation in the case of scalar and $f(R)$-modified cosmology.

\section{A. Scalar cosmology with Lagrange multiplier}

In order to provide a simple realization of cyclicity in this scenario, we start by imposing a desirable form of $H(t)$ that corresponds to a cyclic behavior. We consider a specific, simple, but quite general example, namely we assume a cyclic universe with an oscillatory scale factor of the form

$$
a(t)=A \sin (\omega t)+a_{c},
$$


where we have shifted $t$ in order to eliminate a possible additional parameter standing for the phase ${ }^{1}$. Furthermore, the non-zero constant $a_{c}$ is inserted in order to eliminate any possible singularities from the model. In such a scenario $t$ varies between $-\infty$ and $+\infty$, and $t=0$ is just a specific moment without any particular physical meaning. Finally, note that the bounce occurs at $a_{B}(t)=a_{c}-A$. Straightforwardly we find:

$$
\begin{aligned}
H(t) & =\frac{A \omega \cos (\omega t)}{A \sin (\omega t)+a_{c}} \\
\dot{H}(t) & =-\frac{A \omega^{2}\left[A+a_{c} \sin (\omega t)\right]}{\left[A \sin (\omega t)+a_{c}\right]^{2}} .
\end{aligned}
$$

Concerning the matter content of the universe we assume it to be dust, namely $w_{m} \approx 0$, which inserted in the evolution-equation $\dot{\rho}_{m}+3 H\left(1+w_{m}\right) \rho_{m}=0$ gives the usual dust-evolution $\rho_{m}=\rho_{m 0} / a^{3}$, with $\rho_{m 0}$ its value at present.

Now, we first consider $\phi$ to be a canonical field, that is we choose $\omega(\phi)=1$. Concerning the potential $U(\phi)$ that will give as the solution for $\phi(t)$ according to (9), we choose a simple and easily-handled form, namely

$$
U(\phi)=\frac{m^{4}}{2}
$$

where $m$ is a constant with mass-dimension. In this case, (9) leads to

$$
\phi=m^{2} t
$$

having chosen the + sign in (9). As we have said in subsection $\amalg A$ such a simple relation for $\phi(t)$ allows to replace $t$ by $\phi / m^{2}$ in all the aforementioned relations. Therefore, substitution of (20), (21) and (22) into (12), and using $\phi$ instead of $t$, provides the corresponding expression for $V(\phi)$ that generates such an $H(t)$-solution:

$$
\begin{aligned}
V(\phi)=\frac{m^{4}}{2} & +\frac{1}{\kappa^{2}}\left\{2\left\{-\frac{A \omega^{2}\left[A+a_{c} \sin \left(\omega \frac{\phi}{m^{2}}\right)\right]}{\left[A \sin \left(\omega \frac{\phi}{m^{2}}\right)+a_{c}\right]^{2}}\right\}\right. \\
& \left.+3\left\{\frac{A \omega \cos \left(\omega \frac{\phi}{m^{2}}\right)}{A \sin \left(\omega \frac{\phi}{m^{2}}\right)+a_{c}}\right\}\right\} .
\end{aligned}
$$

Note that in the case of dust matter, the reconstructed potential does not depend on the matter energy density and its evolution. Finally, for completeness, we present

\footnotetext{
${ }^{1}$ Note that if the average of an oscillatory scale factor keeps increasing throughout the evolution, it could yield a recurrent universe which unifies the early time inflation and late time acceleration [24, 25].
}

also the $\lambda(t)$ evolution, which according to (11) reads

$$
\begin{gathered}
\lambda(t)=-1-\frac{2}{\kappa^{2} m^{4}}\left\{-\frac{A \omega^{2}\left[A+a_{c} \sin (\omega t)\right]}{\left[A \sin (\omega t)+a_{c}\right]^{2}}\right\} \\
-\frac{1}{m^{4}} \rho_{m 0}\left\{A \sin (\omega t)+a_{c}\right\}^{-3} .
\end{gathered}
$$

In order to provide a more transparent picture of the obtained cosmological behavior, in Fig. [1 we present the evolution of the oscillatory scale factor (19) and of the Hubble parameter (20), with $A=1, \omega=1$ and $a_{c}=1.3$, where all quantities are measured in units with $8 \pi G=1$.

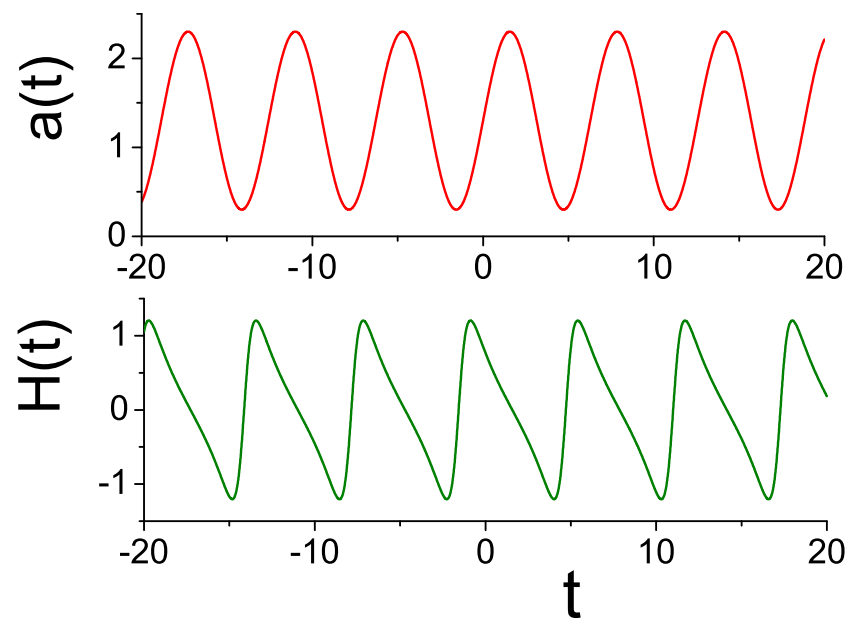

Figure 1: The evolution of the scale factor $a(t)$ and of the Hubble parameter $H(t)$ of the ansatz [19), with $A=1, \omega=1$ and $a_{c}=1.3$. All quantities are measured in units where $8 \pi G=1$.

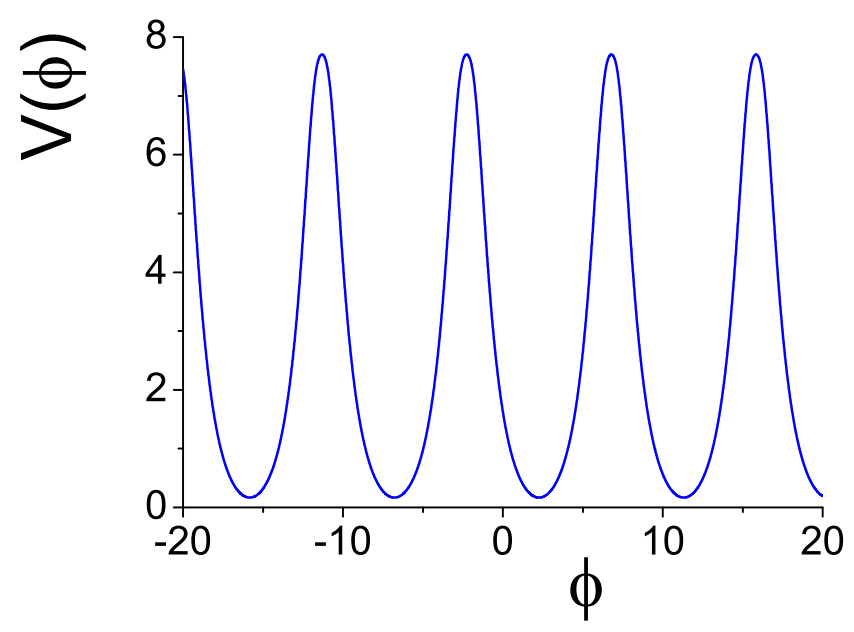

Figure 2: $V(\phi)$ from 24) that reproduces the cosmological evolution of Fig. 1, with $\omega(\phi)=1, U(\phi)=m^{4} / 2$ and $m=1.2$. All quantities are measured in units where $8 \pi G=1$. 
Note that $H(t)$ by construction satisfies the requirements for cyclicity, described in the beginning of this section. Furthermore, in Fig. 2 we depict the corresponding potential $V(\phi)$ given by (24). From these figures we observe that an oscillating and singularity-free scale factor can be generated by an oscillatory form of the scalar potential $V(\phi)$ (although of not a simple function as that of $a(t)$, as can be seen by the slightly different form of $V(\phi)$ in its minima and its maxima). This $V(\phi)$-form was more or less theoretically expected, since a non-oscillatory $V(\phi)$ would be physically impossible to generate an infinitely oscillating scale factor and a universe with a form of timesymmetry. Note also that, having presented the basic mechanism, we can suitably choose the oscillation frequency and amplitude in order to get a realistic oscillation period and scale factor for the universe. Finally, we stress that although we have presented the above specific simple example, we can straightforwardly perform the described procedure imposing an arbitrary oscillating ansatz for the scale factor.

The aforementioned bottom to top approach was enlightening about the form of the scalar potential that leads to a cyclic cosmological behavior. Therefore, one can perform the above procedure the other way around, starting from a specific oscillatory $V(\phi)$ and resulting to an oscillatory $a(t)$, following the steps described in the first part of subsection $1 \mathrm{~A}$. As a specific example we consider the simple case

$$
V(\phi)=V_{0} \sin \left(\omega_{V} \phi\right)+V_{c},
$$

with $U(\phi)$ chosen as in (22) and thus (23) holds too. Despite the simplicity of $V(\phi)$, the differential equation (10) cannot be solved analytically, but it can be easily handled numerically. In Fig. 3 we depict the corresponding solution for $H(t)$ (and thus for $a(t)$ ) under the ansatz (26), with $V_{0}=3, \omega_{V}=1$ and $V_{c}=3$, with $\alpha(0)=3.2$ and $H(0)=-0.7$ (in units where $8 \pi G=1$ ). As expected, we do obtain an oscillatory universe.

\section{B. $f(R)$-gravity with Lagrange multiplier}

In order to provide a simple realization of cyclicity in this scenario, we start by imposing a desirable form of $H(t)$ that corresponds to a cyclic behavior. Due to the complicated structure of the reconstruction procedure, we will choose an easier ansatz comparing to the previous subsection, which allows for the extraction of analytical results. In particular, having described the general requirements for an $H(t)$ that gives rise to cyclic cosmology in the beginning of the present section, we choose $H(t)$ (and not $a(t)$ ) to be straightaway a sinusoidal function, that is

$$
H(t)=A_{H} \sin \left(\omega_{H} t\right)
$$

which gives rise to a scale factor of the form

$$
a(t)=A_{H 0} \exp \left[-\frac{A_{H} \cos \left(\omega_{H} t\right)}{\omega_{H}}\right],
$$

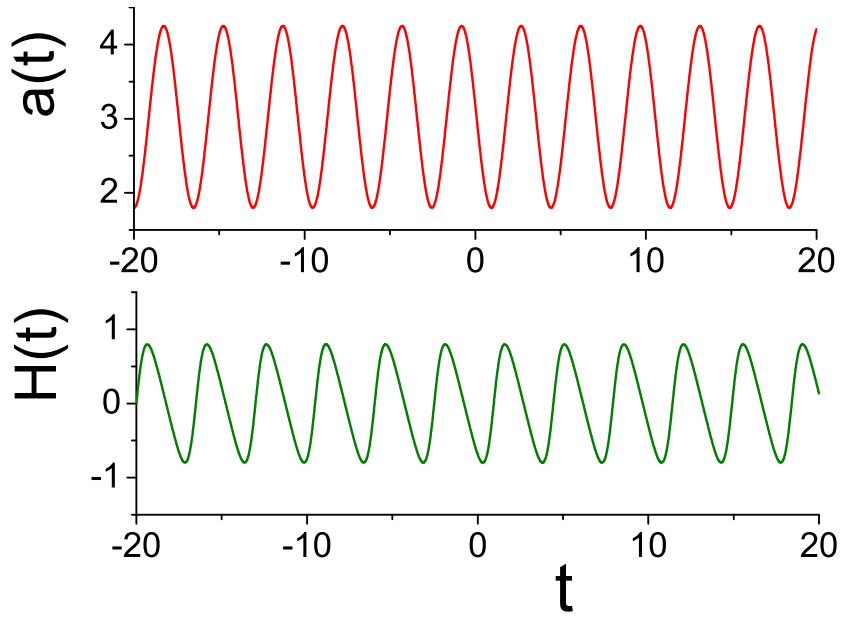

Figure 3: The evolution of the scale factor $a(t)$ and of the Hubble parameter $H(t)$, for a scalar potential of the ansatz (26), with $V_{0}=3, \omega_{V}=1$ and $V_{c}=3, \alpha(0)=3.2$ and $H(0)=-0.7$. All quantities are measured in units where $8 \pi G=1$.

which is oscillatory and always non-zero. Inserting (27) and its derivative into (17) we obtain $R(t)$ as

$$
R(t)=6 A_{H} \omega_{H} \cos \left(\omega_{H} t\right)+12 A_{H}^{2} \sin ^{2}\left(\omega_{H} t\right),
$$

a relation that can be easily inverted giving

$$
t(R)=\frac{1}{\omega_{H}} \arccos \left[\frac{3 \omega_{H}+\sqrt{3\left(48 A_{H}^{2}-4 R+3 \omega_{H}^{2}\right)}}{12 A_{H}}\right],
$$

where we have kept the plus sign in the square root. Finally, $f_{2}(R)$ can be reconstructed using (18), leading to

$$
\begin{aligned}
& f_{2}(R)=-\frac{1}{4} \omega_{H}^{2}\left(48 A_{H}^{2}-4 R+3 \omega_{H}^{2}\right) \\
& \times\left[-2 R+3 \omega_{H}^{2}+\omega_{H} \sqrt{3\left(48 A_{H}^{2}-4 R+3 \omega_{H}^{2}\right)}\right]
\end{aligned}
$$

In summary, such an ansatz for $f_{2}(R)$ produces the cyclic universe with scale factor (28). Note that $f_{2}(R)$ has a remarkably simple form, and this is an advantage of the scenario at hand, since in conventional $f(R)$-gravity one needs very refined and complicated forms of $f(R)$ in order to reconstruct a given cosmological evolution [26]. However, as we stated in the beginning of subsection $\amalg B$ in the $f(R)$-version of Lagrange-modified gravity one cannot incorporate the presence of matter in a convenient way that will allow for an analytical treatment. The absence of matter evolution in a cyclic scenario is a disadvantage, since we cannot reproduce the evolution epochs of the universe. Therefore it would be necessary to construct a formalism that would allow for such a matter inclusion, similarly to the case of Lagrange-multiplier modified scalar-field cosmology. Such a project is in preparation. 


\section{STABILITY ANALYSIS}

A central issue in all cyclic models is the stability analysis of the cosmological perturbations along with background evolution. In Newtonian gauge the linear metric perturbation is given by

$$
d s^{2}=-(1+2 \Phi) d t^{2}+a^{2}(t)(1-2 \Psi) d \mathbf{x}^{2}
$$

where the variable $\Phi$ is the so-called Newtonian potential which describes the scalar part of metric perturbation. In the following we will study the evolution of the Newtonian potential near the bouncing point, for the two above scenarios respectively.

\section{A. Scalar cosmology with Lagrange multiplier}

An explicit analysis on the Newtonian potential of the scenario of scalar cosmology with Lagrange multiplier was performed in [21]. A very remarkable feature of this model is that the system possesses no wavelike modes and thus the sound speed of perturbations is identically zero in all backgrounds. By virtue of this particular property, it could be possible to control the dangerous growth of unstable modes of cosmological perturbations in the contracting phases of the cyclic universe.

Assuming that there is no anisotropic stress in our model, then $\Psi=\Phi$. Consequently, we only have one degree of freedom of cosmological perturbations. According to the analysis of [21], one deduces that when the background evolution is dominated by the scalar field $\phi$, the perturbation equation of the Newtonian potential can be expressed as

$$
\Phi^{\prime \prime}+\left(1-\frac{H^{\prime \prime}}{H^{\prime}}\right) \Phi^{\prime}+\left(\frac{H^{\prime}}{H}-\frac{H^{\prime \prime}}{H^{\prime}}\right) \Phi \simeq 0,
$$

where the prime denotes the derivative with respect to $\ln a$. Note that the dust-like sound speed $c_{s}=0$ has been applied to eliminate the gradient term. Equation (32) yields the generic solution [21]

$$
\Phi \simeq D(x)\left(1-\frac{H}{a} \int^{a} \frac{d a}{H}\right)+S(x) \frac{H}{a},
$$

which is applicable in all scales. Thus, in this solution there are two modes $D(x)$ and $S(x)$, which are arbitrary functions of spatial coordinates, and their explicit forms can be determined by certain boundary conditions.

Observing the second term of the right-hand-side of (33), one may concern that the Newtonian potential might be divergent on the bouncing point, when $H=0$. Fortunately, this does not happen in a generic bounce model. As it was shown in phenomenological studies of generic bounce scenarios [27], one can impose an approximate parametrization of the Hubble parameter as a linear function of the cosmic time, that is $H=\xi t$, nearby the bouncing point $t_{B}=0$, with $\xi$ being a positive constant. Doing so, in the neighborhood of a bounce in a specific cycle the Newtonian potential can be solved as

$$
\Phi_{B} \simeq D\left[1-\sqrt{\frac{\pi}{2}} \xi t e^{-\frac{\xi t^{2}}{2}}\left(e^{\frac{\xi t^{2}}{2}}-1\right)^{\frac{1}{2}}\right]+S \frac{\xi t}{a_{B}} e^{-\frac{\xi t^{2}}{2}}
$$

where $a_{B}$ is the value of the scale factor at the bouncing point.

Relation 34 presents convergent behavior, and thus the perturbations are able to pass through the bounce smoothly and without any pathology. Therefore, the scenario at hand indeed provides a satisfactory way to realize a cyclic picture without instability on its perturbations.

\section{B. $f(R)$-gravity with Lagrange multiplier}

When we are dealing with $f(R)$-gravity with Lagrange multiplier, we cannot use the simplifying relation $\Psi=$ $\Phi$, even under the assumption of zero anisotropic stress. Therefore, in order to study the number of degrees of freedom in this case it is convenient to expand the action into quadratic order, which can be formally expressed as

$$
S^{(2)}=\int d^{4} x \sqrt{-g}\left[\frac{f_{1, R R}}{2} \delta_{1} R^{2}+f_{1}\left(-\frac{g_{\mu \nu}}{2} \delta g^{\mu \nu} \delta_{1} R+\delta_{2} R\right)+\left(\frac{g_{\mu \nu}}{2} \delta g^{\mu \nu}\right) \lambda C_{2}\right]
$$

where $\delta_{1} R$ and $\delta_{2} R$ denote the perturbed Ricci scalar at first and second order respectively. Moreover, $C_{2}$ is the perturbed constraint equation at first order, which form is given by

$$
\frac{1}{2} \delta g^{\mu \nu} \partial_{\mu} R \partial_{\nu} R+\partial_{\mu} R \partial^{\mu} \delta_{1} R+f_{2, R} \delta_{1} R=0
$$


In principle, one could worry since the above action involves two scalar modes and higher derivative terms, which could imply instabilities. However, this is not the case since the higher derivative terms can be fixed by the perturbed constraint equations. In addition, the vanishing of the $(i, j)$ component of the perturbed Einstein equation allows to eliminate one degree of freedom. Therefore, there is still only one mode of metric perturbation which is able to propagate freely.

In the following, we would like to focus on the propagation of the metric perturbation around the bouncing point, which is crucial to the stability analysis of a cyclic scenario. For calculation convenience we assume that the bounce takes place slowly, and then the universe approaches a static phase around the bounce asymptotically. Under this assumption we can obtain the kinetic terms of the perturbation equation, which take the following approximate form:

$$
\ddot{Q}-\frac{\nabla^{2}}{a^{2}} Q+\frac{f_{1}}{f_{1, R}} Q+\ldots=0,
$$

in which $Q \equiv \Phi+\Psi$. Although this equation is far from a complete form, one can still extract a few important issues. Namely, the sound speed of the perturbation is unity under the assumption of slow bounce, as it can be read from the coefficient before the gradient term. Furthermore, we confirm that there exist only a single degree of freedom in the $f(R)$ cosmology with Lagrange multiplier.

We mention here that we investigated the stability only under the assumption of slow bounce. And the result in this case illustrates that it is possible for the cosmological perturbation to evolve through the bounces. However, it is necessary to point out that a more complete and generic analysis should be performed in order to constrain the parameter space of the scenario. Such a general analysis is left for future investigation.

\section{CONCLUSIONS}

In this work we investigated cyclic evolutions in a universe governed by Lagrange-multiplier modified gravity. In order to be more general we considered the scenario of modified gravity through Lagrange multipliers in both scalar-field cosmology, as well as in $f(R)$ one.

In the case of scalar cosmology, the use of a second field that is the Lagrange multiplier of the usual scalar, leads to a rich cosmological behavior. In particular, one can obtain an arbitrary cyclic evolution for the scale factor, by reconstructing suitably the scalar potential. As expected, an oscillatory scale factor is induced by an oscillatory potential, and we were able to perform such a procedure starting either from the scale factor or from the potential. Additionally, the matter sector can be also incorporated easily, and this is an advantage of the scenario, since it allows for the successful reproduction of the thermal history of the universe.

In the case of $f(R)$-gravity, we considered a Lagrange multiplier field and a second form $f_{2}(R)$. This scenario leads also to a rich behavior, and one can acquire cyclic cosmology by suitably reconstructing $f_{2}(R)$, which proves to be of a very simple form, contrary to the conventional $f(R)$-gravity. However, the complexity of the model does not allow for the easy incorporation of the matter sector, since one cannot extract analytical results, and this is a disadvantage of the construction.

In summary, we saw that Lagrange-multiplier modified gravity may lead to cyclic behavior very easily, and the scenario is much more realistic in the case of scalar cosmology. Since a necessary test of every cosmological scenario is to examine the evolution of perturbations through the bounce [28], we extended our analysis beyond the background level, in both scenarios we considered. For the case of scalar cosmology the perturbation behaves as a frozen mode without oscillations, since its sound speed is vanishing. For the case of $f(R)$-gravity and under the assumption of slow bounce, we obtain a dynamical degree of freedom of which the sound speed is almost unity. Thus, in conclusion, it is possible for the cosmological perturbation to evolve through the bounces. However, a more generic perturbation analysis, beyond the slow bounce assumption, is needed, but since it lies beyond the scope of this work it is left for future investigation.

\section{Acknowledgments}

It is a pleasure to thank the anonymous referees for valuable comments. The work of YFC is supported in part by the Arizona State University Cosmology Initiative.
[1] A. H. Guth, Phys. Rev. D 23, 347 (1981); A. D. Linde, Phys. Lett. B 108, 389 (1982); A. J. Albrecht and P. J. Steinhardt, Phys. Rev. Lett. 48, 1220 (1982).

[2] A. Borde and A. Vilenkin, Phys. Rev. Lett. 72, 3305 (1994) arXiv:gr-qc/9312022.

[3] V. F. Mukhanov and R. H. Brandenberger, Phys. Rev.
Lett. 68, 1969 (1992).

[4] G. Veneziano, Phys. Lett. B 265, 287 (1991); M. Gasperini and G. Veneziano, Astropart. Phys. 1, 317 (1993) arXiv:hep-th/9211021.

[5] J. Khoury, B. A. Ovrut, P. J. Steinhardt and N. Turok, Phys. Rev. D 64, 123522 (2001) arXiv:hep-th/0103239; 
J. Khoury, B. A. Ovrut, N. Seiberg, P. J. Steinhardt and N. Turok, Phys. Rev. D 65, 086007 (2002) arXiv:hep-th/0108187.

[6] R. Brustein and R. Madden, Phys. Rev. D 57, 712 (1998) arXiv:hep-th/9708046. T. Biswas, A. Mazumdar and W. Siegel, JCAP 0603, 009 (2006) arXiv:hep-th/0508194]; T. Biswas, R. Brandenberger, A. Mazumdar and W. Siegel, JCAP 0712, 011 (2007) arXiv:hep-th/0610274.

[7] Y. Shtanov and V. Sahni, Phys. Lett. B 557, 1 (2003) arXiv:gr-qc/0208047; E. N. Saridakis, Nucl. Phys. B 808, 224 (2009) arXiv:0710.5269 [hep-th].

[8] Y. F. Cai and E. N. Saridakis, JCAP 0910, 020 (2009) arXiv:0906.1789 [hep-th]]; E. N. Saridakis, Eur. Phys. J. C 67, 229 (2010) arXiv:0905.3532 [hep-th]].

[9] M. Bojowald, Phys. Rev. Lett. 86, 5227 (2001) arXiv:gr-qc/0102069.

[10] J. Martin and P. Peter, Phys. Rev. D 68, 103517 (2003) arXiv:hep-th/0307077.

[11] Y. F. Cai, T. Qiu, Y. S. Piao, M. Li and X. Zhang, JHEP 0710, 071 (2007) arXiv:0704.1090 [gr-qc]l; Y. F. Cai, T. Qiu, R. Brandenberger, Y. S. Piao and X. Zhang, JCAP 0803, 013 (2008) arXiv:0711.2187 [hep-thl]; Y. F. Cai, E. N. Saridakis, M. R. Setare and J. Q. Xia, arXiv:0909.2776 [hep-th].

[12] Y. F. Cai and X. Zhang, JCAP 0906, 003 (2009) arXiv:0808.2551 [astro-ph]]; Y. F. Cai, T. t. Qiu, J. Q. Xia and X. Zhang, Phys. Rev. D 79, 021303 (2009) arXiv:0808.0819 [astro-ph]]; Y. F. Cai, W. Xue, R. Brandenberger and $X$. Zhang, JCAP 0905, 011 (2009) arXiv:0903.0631 [astro-ph.CO]]; Y. F. Cai, W. Xue, R. Brandenberger and X. m. Zhang, JCAP 0906, 037 (2009) arXiv:0903.4938 [hep-th]]; Y. F. Cai and X. Zhang, Phys. Rev. D 80, 043520 (2009) arXiv:0906.3341 [astro-ph.CO]].

[13] E. N. Saridakis and J. Ward, Phys. Rev. D 80, 083003 (2009) arXiv:0906.5135 [hep-th]]; E. N. Saridakis and S. V. Sushkov, Phys. Rev. D 81, 083510 (2010) arXiv:1002.3478 [gr-qc]]; E. N. Saridakis and J. M. Weller, Phys. Rev. D 81, 123523 (2010) arXiv:0912.5304 [hep-th]].

[14] R. C. Tolman, Relativity, Thermodynamics and Cosmology, Oxford U. Press (1934).

[15] P. J. Steinhardt and N. Turok, Phys. Rev. D 65, 126003 (2002) arXiv:hep-th/0111098; P. J. Steinhardt and N. Turok, Science 296, 1436 (2002).

[16] H. H. Xiong, Y. F. Cai, T. Qiu, Y. S. Piao and X. Zhang,
Phys. Lett. B 666, 212 (2008) arXiv:0805.0413 [astro$\mathrm{ph}]$.

[17] J. E. Lidsey, D. J. Mulryne, N. J. Nunes and R. Tavakol, Phys. Rev. D 70, 063521 (2004) arXiv:gr-qc/0406042; Y. S. Piao and Y. Z. Zhang, Nucl. Phys. B 725, 265 (2005) arXiv:gr-qc/0407027]; Y. S. Piao, Phys. Rev. D 70, 101302 (2004) arXiv:hep-th/0407258]; H. H. Xiong, T. Qiu, Y. F. Cai and X. Zhang, Mod. Phys. Lett. A 24, 1237 (2009) arXiv:0711.4469 [hep-th]]; X. Zhang, Eur. Phys. J. C 60, 661 (2009) arXiv:0708.1408 [gr-qc]]; T. Biswas and S. Alexander, Phys. Rev. D 80, 043511 (2009) arXiv:0812.3182 [hep-th]]; Y. S. Piao, Phys. Lett. B 677, 1 (2009) arXiv:0901.2644 [gr-qc]].

[18] S. Mukherji and M. Peloso, Phys. Lett. B 547, 297 (2002) arXiv:hep-th/0205180; J. Khoury, P. J. Steinhardt and N. Turok, Phys. Rev. Lett. 92, 031302 (2004) arXiv:hep-th/0307132 ; L. Baum and P. H. Frampton, Phys. Rev. Lett. 98, 071301 (2007) arXiv:hep-th/0610213.

[19] M. Novello and S. E. P. Bergliaffa, Phys. Rept. 463, 127 (2008) arXiv:0802.1634 [astro-ph]].

[20] R. H. Brandenberger, V. F. Mukhanov and A. Sornborger, Phys. Rev. D 48, 1629 (1993) arXiv:gr-qc/9303001.

[21] E. A. Lim, I. Sawicki and A. Vikman, JCAP 1005, 012 (2010) arXiv:1003.5751 [astro-ph.CO]].

[22] S. Capozziello, J. Matsumoto, S. Nojiri and S. D. Odintsov, Phys. Lett. B 693, 198 (2010) arXiv:1004.3691 [hep-th].

[23] C. Gao, Y. Gong, X. Wang and X. Chen, arXiv:1003.6056 [astro-ph.CO]; S. Nojiri and S. D. Odintsov, Phys. Lett. B 691, 60 (2010) arXiv:1004.3613 [hep-th]].

[24] B. Feng, M. Li, Y. S. Piao and X. Zhang, Phys. Lett. B 634, 101 (2006) arXiv:astro-ph/0407432.

[25] Y. F. Cai, J. Liu and H. Li, Phys. Lett. B 690, 213 (2010) arXiv:1003.4526 [astro-ph.CO]].

[26] S. Nojiri and S. D. Odintsov, J. Phys. Conf. Ser. 66, 012005 (2007) arXiv:hep-th/0611071.

[27] Y. F. Cai, T. t. Qiu, R. Brandenberger and X. m. Zhang, Phys. Rev. D 80, 023511 (2009) arXiv:0810.4677 [hepth]].

[28] R. H. Brandenberger, Phys. Rev. D 80, 023535 (2009) arXiv:0905.1514 [hep-th]]; J. Zhang, Z. G. Liu and Y. S. Piao, arXiv:1007.2498 [hep-th]; T. Qiu and K. C. Yang, arXiv:1007.2571 [astro-ph.CO]. 